\title{
INTEGRAL monitoring of NGC 4151: Evidence for a truncated accretion disk?
}

\author{
Stéphane Paltani ${ }^{1}$, P. Lubiński ${ }^{2}$, A.A. Zdziarski ${ }^{3}$, R. Walter ${ }^{1}$, V. Beckmann ${ }^{4}$, S. \\ Soldi $^{5}$, C. Ferrigno ${ }^{1}$, T. J.-L. Courvoisier ${ }^{1}$ \\ ${ }^{1}$ ISDC Data Centre for Astrophysics, Observatory of the University of Geneva, Switzerland \\ ${ }^{2}$ Centrum Astronomiczne im. M. Kopernika, Toruń, Poland \\ ${ }^{3}$ Centrum Astronomiczne im. M. Kopernika, Warszawa, Poland \\ ${ }^{4}$ APC, UMR 7164, Université Paris 7 Denis Diderot, France \\ ${ }^{5}$ Laboratoire AIM - CNRS - CEA/DSM - Université Paris Diderot, France \\ E-mail: Stephane.Paltani@unige.ch
}

\begin{abstract}
We present some results of the INTEGRAL observation campaign of the bright Seyfert galaxy NGC 4151. The INTEGRAL observations cover 7 years, for a total of more than 1.5Ms effective exposure with ISGRI over 31 revolutions. We determine the physical parameters of the plasma responsible for the medium and hard X-ray emission $(>3 \mathrm{keV})$. Significant variability in plasma temperature and optical depth are found, but the Compton $y$ parameter is practically constant, indicating a stable geometry. The plasma is found to be photon-starved, with a Comptonized flux about 15 times the seed-photon flux. We measure the reflected fraction and conclude that both a distant and a nearby reflector are needed, with similar reflection parameters, which we interpret as originating from the torus and from the accretion disk, respectively. The small disk reflection parameter, $R_{\text {Disk }} \simeq 0.27$, is an evidence for a truncated accretion disk, turning into a hot, radiatively inefficient accretion flow at a radius determined from the soft-photon seed emission to be $\sim 18 R_{\mathrm{G}}$.
\end{abstract}

8th INTEGRAL Workshop "The Restless Gamma-ray Universe”- Integral2010,

September 27-30, 2010

Dublin Ireland 


\section{Introduction}

Hard X-ray emission of Seyfert galaxies typically consists of two components: the direct component, which is generally represented as a cut-off power-law with an index $\Gamma \simeq 1.9$ [2] and a component which appears as a hump around $30 \mathrm{keV}$ and which is assumed to result from the reflection of the direct component on surrounding cold material [5]. These two components are therefore closely linked, and this connection, if properly investigated, could provide important clues about the geometry of the system.

The most probable origin of the direct component is through Comptonization of soft photons, probably originating from the accretion disk, in a plasma located in the vicinity of the soft-photon source in a still rather unconstrained geometry. Comptonization models have been available for quite some time [9, 6]; however, adequate hard X-ray data are still very scarce, and the study of plasma parameters could only be made for few sources [10]. Thermal Comptonization in a hot plasma explains nicely the spectral features, with typical parameters determined for 11 sources being temperatures $\mathrm{k} T_{e} \sim 80 \mathrm{keV}$ and optical depths $\tau \sim 1.7$ [10].

Reflection of hard X-ray emission from the hot plasma may take place in the accretion disk, but also in a distant torus [3], and the hard X-ray emission of AGN may in principle present signatures of the distribution of the cold material. Again, very good-quality hard X-ray spectra are needed in order to provide constraints on the AGN geometry.

In this work, we present the results from an INTEGRAL monitoring campaign on NGC 4151, the brightest Seyfert galaxy in hard X-rays. The goal of the campaign is to constrain the emission mechanisms in the hard X-rays, in particular taking advantage of the variability of the source on the long time scales investigated by these observations. This paper presents a summary of an in-depth analysis of the INTEGRAL observations; the full analysis is published in [4].

\section{Data}

\subsection{The INTEGRAL campaign}

NGC 4151 has been observed many times with INTEGRAL, either during good-quality dedicated observations in May 2003, January, May and December 2007 and May 2008, or within observations of nearby objects. In the latter case, the off-axis angles are in the range $9-15^{\circ}$, which reduces significantly the effective exposure times. All available INTEGRAL data have been reduced with OSA 7.0 with default parameters (OSA 8.0 in the case of JEM-X). Figure 1 shows the INTEGRAL/ISGRI $20-100 \mathrm{keV}$ light curve. The light curve is completed with fluxes from previous X-ray observatories, in particular from BATSE and OSSE on board Compton-GRO and from BeppoSAX/PDS. Except for a few, probably rather uncertain measurements dated before 1980, the INTEGRAL campaign is found to cover the whole range of historical $20-100 \mathrm{keV}$ fluxes over almost 7 years.

In order to build good-quality hard X-ray spectra, we group the different INTEGRAL data sets according to their fluxes. We define four "states" according to the $20-100 \mathrm{keV}$ flux, which we call "bright", "medium-bright", "medium" and "dim", respectively. Most INTEGRAL data are in the dim state, so that the dim-state and the bright-state spectra have the best signal-to-noise ratio. 

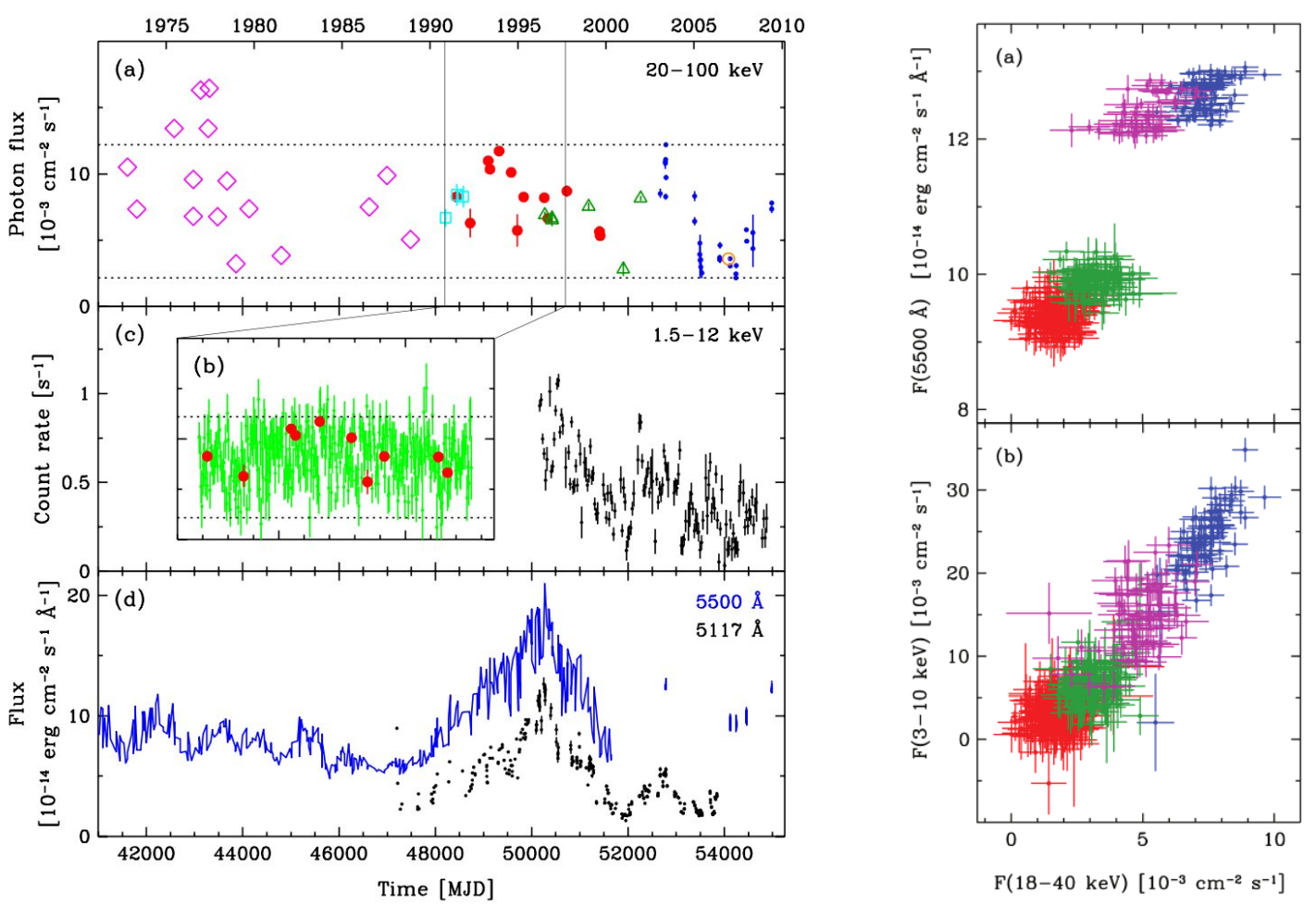

Figure 1: Left: Historical NGC 4151 light curves. (a) Hard X-rays (20-100 keV). The blue points show the INTEGRAL/ISGRI observations. The green, red, cyan and magenta points are, respectively, from BeppoSAX, OSSE, Sigma and various sources. (b) C-GRO/BATSE monitoring. (c) R-XTE/ASM monitoring. (d) Optical monitoring from [1] (blue points), INTEGRAL/OMC (isolated blue points) and [8] (black points). Right: OMC $5500 \AA$ flux (a) and JEM-X 3-10 keV flux (b) versus ISGRI 18-40 keV flux. The colours identify the three main states, with, additionally, Revs. 0076, 0809-0811 in magenta.

Although the (especially JEM-X) fluxes have significant statistical uncertainties, Fig. 1 shows that the ISGRI fluxes are very well linearly correlated with the JEM-X $3-10 \mathrm{keV}$ ones. A correlation is also observed with the optical OMC data, but there is evidence for another variability mechanism in the optical which is uncorrelated with the X-ray emission and possibly operating on long time scales.

\subsection{Additional X-ray data}

In order to study the broad-band emission, we collected all RXTE, XMM-Newton and Suzaku observations of NGC 4151 which are more or less contemporaneous with the INTEGRAL observations. Selection of low-energy spectra from other satellites was based on Swift/BAT fluxes measured at a time close to those observations, and then compared to the ISGRI flux for a given state. Selection using JEM-X flux can be done in principle, but it will be affected by variable absorption and the rather high uncertainty of JEM-X fluxes. We associate this way three XMM-Newton and one RXTE observations to the bright state, one XMM-Newton, one Suzaku and two RXTE observations to the dim state, and one XMM-Newton observation to the medium state. Details abound X-ray observations can be found in Table 2 of [4]. In the spectral analysis, we remove the 


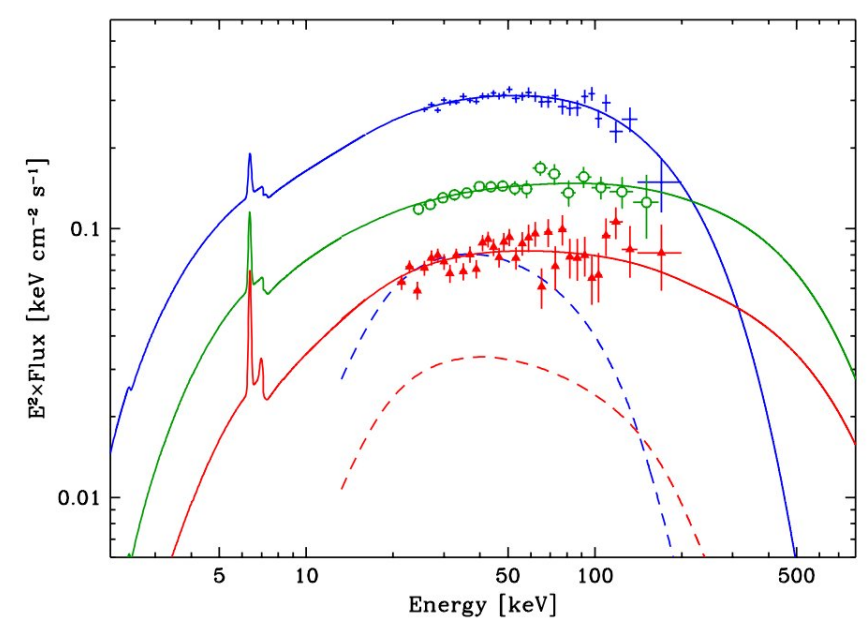

Figure 2: Comptonization models fitted to the bright (blue), medium (green) and dim (red) states for a plasma in a spherical geometry. Only the INTEGRAL/ISGRI data are shown for clarity. The reflection components are shown as a dashed line for the bright and dim states.

data points below $2.5 \mathrm{keV}$ in order to alleviate the effects of the complex, variable absorption in NGC 4151 [7].

\section{Broad-band spectral modeling}

We model the X-ray emission of NGC 4151 with a physically-motivated model of thermal Comptonization of soft photons from the accretion disk [6] assuming different geometries (sphere, cylinder, slab, hemisphere) and a disk characteristic temperature fixed to $10 \mathrm{eV}$. Figure 2 shows the broad-band X-ray spectra models for a spherical geometry for the bright, medium and dim states, with only the INTEGRAL/ISGRI data represented for clarity. The fits are all formally acceptable, with reduced- $\chi^{2}<1$. In addition, in the bright state thermal Comptonization models significantly improve the fits compared to a simpler cut-off power-law model. The high-energy cut-off (and therefore plasma temperature) is markedly lower in the bright state than in the two other states. The spectral slopes are however rather similar.

The bright state is characterized by a low temperature $\left(\mathrm{k} T_{\mathrm{e}} \sim 54-73 \mathrm{keV}\right)$ and an optical depth $\tau \sim 1.3-2.6$, depending on geometry. All the different geometries produce formally acceptable fits, with little significant difference. In the dim state, the plasma temperature is found to be $\mathrm{k} T_{\mathrm{e}} \sim 186-227 \mathrm{keV}$ with smaller optical depths $\tau \sim 0.31-0.68$. Again the geometry cannot be adequately constrained by the fits. The medium state is less well constrained, but parameters are found to have intermediate values of $\mathrm{k} T_{\mathrm{e}} \sim 100-200 \mathrm{keV}$ for the temperature and $\tau \sim 0.7-1.5$ for the optical depth (assuming a spherical geometry).

The absolute normalization of the reflection component is about twice as large in the case of the bright state than in the dim state. The reflection fraction $R$ is found to lie between 0.75 and 1.04 in the dim state and between 0.38 and 0.55 in the bright state, while it is not formally required in the medium state. This implies a change in absolute reflection intensity by a factor $\sim 2.5$ while 
the continuum has changed by a factor $\sim 4$. The significant variability of the reflection indicates the presence of a nearby reflector, whose reflected emission follows closely the continuum. On the other hand, the smaller reflection parameter in the bright state is evidence for the presence of a second, distant reflector, whose response to the primary emission is averaged.

\section{Discussion and conclusions}

\subsection{Reflection fraction}

To explain the variability of the reflected component, we decompose the reflector into a distant, constant one (assumed to be the torus) and a close one (assumed to be the accretion disk), which follows the incident continuum. Assuming constant solid angle for the two reflectors, the reflection fractions $R_{\text {Torus }}$ and $R_{\text {Disk }}$ for both reflectors are constant, hence we have for each state:

$$
F_{\text {Total }}^{S}=F_{\text {Disk }}^{S}+F_{\text {Torus }}
$$

and

$$
F_{\text {Disk }}^{S}=F_{\text {Total }}^{S}\left(R_{\text {Disk }} / R_{\text {Total }}\right),
$$

where $S$ denotes the state (either "bright" or "dim").

Solving these two equations in the two states for $F_{\text {Disk }}$ (bright and $\left.\operatorname{dim}\right), F_{\text {Torus }}$ and $R_{\text {Disk }}\left(F_{\text {Total }}\right.$ and $R_{\text {Total }}$ are measured), we find $R_{\text {Disk }}=0.27$. The reflection fraction due to the torus is then $R_{\text {Torus }}=0.24$. The fact that $R_{\text {Disk }}$ is significantly below 1 means that the accretion disk does not cover a full half-sky as seen from the X-ray source(s). A possible explanation is that the accretion disk is truncated. The Eddington ratio in NGC 4151 is as low as $\sim 0.01-0.02$, which may imply the transition from a standard thin disk into an advection-dominated accretion flow in the central region, effectively removing a fraction of the disk reflector.

\subsection{Plasma parameters and source geometry}

Figure 3 shows the plasma parameters for three different geometries in the $\mathrm{k} T_{\mathrm{e}}$ vs Compton parameter $y$ (left) and $\mathrm{k} T_{\mathrm{e}}$ vs optical depth $\tau$. Very significant changes in plasma temperatures are observed (factor $\sim 3.5$ ), with very small change in spectral index. This is reflected in the roughly constant Compton parameter $y$ in each geometry. The optical depth must therefore vary as the inverse of the temperature. This could happen if the optical depth is proportional to the accretion rate, and the temperature is determined by the energy balance with a constant amplification.

The ratio between power emitted by the plasma and the soft-photon power absorbed by the plasma is roughly constant at a value about 15 , i.e. the plasma is photon-starved. This is consistent with the evidence of a truncated accretion disk found in the variability of the reflected component, as this reduces the solid angle of the soft-photon source as seen by the plasma.

Calculating the soft-photon flux from the normalization of the disk component in the fit, one obtains an inner radius $R_{\mathrm{in}} \simeq 18 \mathrm{R}_{\mathrm{G}}$ for a peak temperature $\mathrm{k} T_{\mathrm{bb}}=5 \mathrm{eV}$, consistent with the existence of a hot inner accretion flow. This is close to the radius above which half of the gravitational energy dissipation takes place in an accretion disk extending from the innermost stable orbit to infinity. In the case of a spherically distributed plasma, this inner radius provides a geometrical estimate of the expected disk reflection fraction $R_{\text {Disk }} \simeq 0.29$, very close to the observed value $R_{\text {Disk }}=0.27$. 


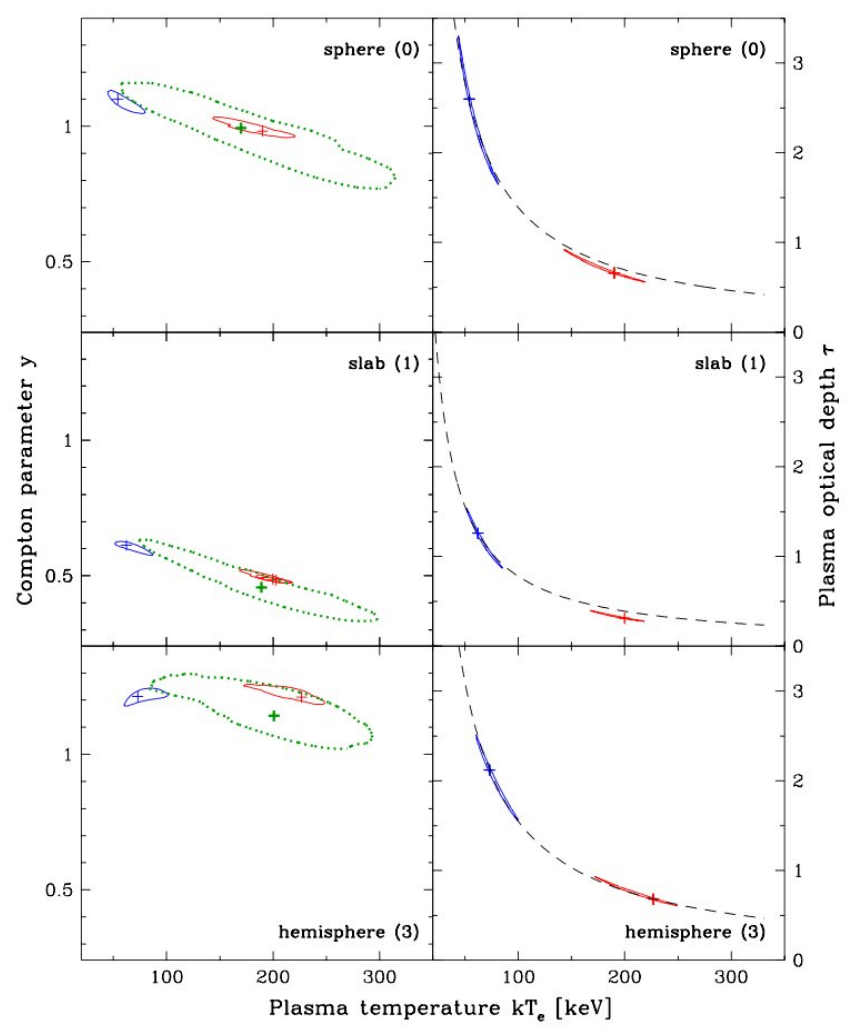

Figure 3: $90 \%$ confidence intervals for the $y$ parameter (left) and optical depth $\tau$ (right) as a function of plasma temperature for the bright (blue), medium (green) and dim (red) states. The dashed lines show the loci of constant $y$ parameters.

\section{References}

[1] Czerny, B., Doroshenko, V. T., Nikołajuk, M., Schwarzenberg-Czerny, A., Loska, Z., \& Madejski, G. 2003, MNRAS, 342, 1222

[2] Dadina, M. 2008, A\&A, 485, 417

[3] Krolik, J. H., Madau, P., \& Zycki, P. T. 1994, ApJL, 420, L57

[4] Lubiński, P., Zdziarski, A. A., Walter, R., Paltani, S., Beckmann, V., Soldi, S., Ferrigno, C., \& Courvoisier, T. 2010, MNRAS, 408, 1851

[5] Magdziarz, P. \& Zdziarski, A. A. 1995, MNRAS, 273, 837

[6] Poutanen, J. \& Svensson, R. 1996, ApJ, 470, 249

[7] Puccetti, S., Fiore, F., Risaliti, G., Capalbi, M., Elvis, M., \& Nicastro, F. 2007, MNRAS, 377, 607

[8] Shapovalova, A. I., Popović, L. Č., Collin, S., Burenkov, A. N., Chavushyan, V. H., Bochkarev, N. G., Benítez, E., Dultzin, D., Kovačević, A., Borisov, N., Carrasco, L., León-Tavares, J., Mercado, A., Valdes, J. R., Vlasuyk, V. V., \& Zhdanova, V. E. 2008, A\&A, 486, 99

[9] Titarchuk, L. 1994, ApJ, 434, 570

[10] Zdziarski, A. A., Poutanen, J., \& Johnson, W. N. 2000, ApJ, 542, 703 